\title{
Analytical and Numerical Study on Irradiate Ignition of Solid Combustibles in Sub-Atmospheric Pressure*
}

\author{
Yuji NAKAMURA** \\ **Hokkaido University, \\ N13 W8, Kita-ku, Sapporo 060-8628 Japan \\ E-mail: yuji-mg@eng.hokudai.ac.jp**
}

\begin{abstract}
The aim of the present study is to understand the basics of spontaneous ignition of irradiated solid combustibles in sub-atmospheric pressure via analytical and numerical approaches. Final goal of this work is to provide the universal description to the recently-observed ignition characters in sub-atmospheric pressure. An irradiated ignition of horizontally-placed, thin cellulose paper in sub-atmospheric pressure is considered here; the sample is heated at the bottom to lead ignition below the surface in gravimetric environment. Two analytical solutions based on classical ignition theories are referred and 3-D numerical simulation is employed for further discussions. Analytical solutions suggest that the observed ignition trend by authors recently is similar to what would be attained in pure diffusion field, not in the stagnation-point flow field which includes the flow-associated heat loss, except near the lowest pressure range to be ignited. According to the 3-D numerical simulation, it is revealed that either pure diffusion or stagnation-point flow field appears depending on the imposed conditions. When longer ignition delay time is expected, an transport process comes to play a role and ignition is close to what would be attained in stagnation-point flow field, rather than the pure diffusion field. Classical ignition theories are useful to understand the insight of ignition processes in low pressure.
\end{abstract}

Key words: Low Pressure, Ignition, Fire

\section{Introduction}

\subsection{Background and Motivation}

Understanding of fire chance in sub-atmospheric pressure is one of essential tasks in space safety management, since the safety is a critical issue in every space mission. In reality, low gravity as well as sub-atmospheric pressure is the typical atmosphere for the conventional space habitats [e.g. 1]. The reason to use the lower pressure is quite simple: we can reduce the pressure difference between inside/outside the habitats artificially. This contributes to simplify the structure of the habitats, since the pressure difference drives main mechanical stress on the structure. In addition, the construction cost could be deduced and more degree of freedom in mechanical design is allowed.

Nonetheless, there is only a few works on fire in sub-atmospherics pressure as compared to those in microgravity. More importantly, there seems no concrete evidence according to the pressure-depended fire characters. Some earlier studies show that an ignitability of the solid (i.e. occurrence of fire) in sub-atmospheric pressure is reduced as compared to that in the standard pressure [2]-[5]. By this fact, it can be considered that we 
have less fire chance in sub-atmospheric pressure. In the recent experiment led by author's group, however, completely opposite trend were notified: the required partial pressure of oxygen (denote " $\mathrm{ppO}_{2}$ " hereafter) was almost linearly reduced as the pressure decreases [6]-[8]. Most recent results are shown in Fig. $1^{\#}$. When the human activity is taken into account in the space, certain amount of $\mathrm{ppO}_{2}$ is required for biological reason. If the above-mentioned "unfamiliar" ignition trend is true, human-required $\mathrm{ppO}_{2}$ could be no longer "safe" in particular range of sub-atmospheric pressure. Overall, we need to gain fundamental understandings on ignition in low pressure.
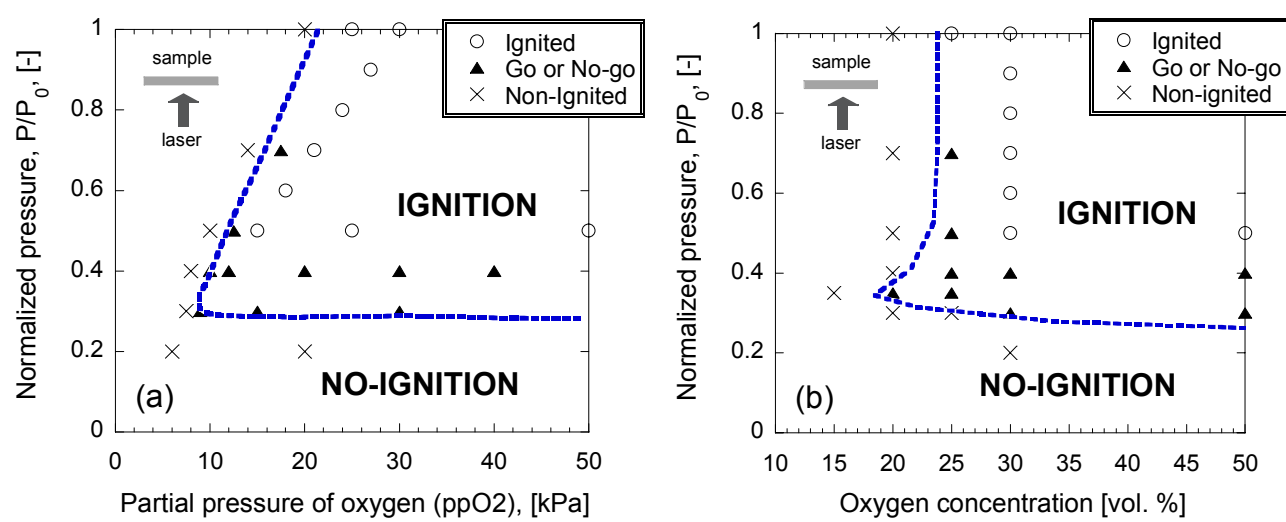

Fig. 1 Experimentally-obtained ignition map in non-dimensionalized pressure $\left(\mathbf{P} / \mathbf{P}_{0}\right)$ vs. oxygen plane. Different expressions of oxygen content are used in (a) and (b) ((a) partial pressure, (b) concentration). Note that $P_{0}$ indicates the standard pressure $(101 \mathrm{kPa})$.

\subsection{Scope and Aim of Present Study}

Ignition is basically time-dependent phenomenon and led by the locally-heated fuel-oxidizer mixture. Since it is difficult to obtain such time- and spatial-variations of all physical properties experimentally, a theoretical approach is selected in this study. Both 1-D analytical and 3-D numerical approaches are employed to explain reasonably well of the experimentally-obtained ignition as seen in Fig.1 and make further discussions on the pressure-depended ignition characters.

Followings are the known mechanism of the irradiate ignition of cellulosic paper considered here [e.g. 9]. Once the solid reaches its decomposed temperature with external energy input, hot gaseous products (including combustible component like carbon monoxide and other hydrocarbons) are evolved due to degradation (pyrolysis/oxidation) reactions and mix with ambient oxygen to form the (hot) combustible mixture. The mixture is further heated by the conduction from the continuously-heated surface to lead the thermal explosion, i.e. ignition, in gas phase. To predict the precise ignition processes, it is necessary to consider the chemical processes in gas/solid phases (exothermic reaction in gas

\footnotetext{
\# In this experiment, thin cellulosic paper (thickness of $0.2 \mathrm{~mm}$ ) is considered as combustible solid and $\mathrm{CO}_{2}$ laser heating is employed from the bottom to the beneath of the sample. Thermal expansion associated with the flash emission is detected and is used to determine if an ignition is attained or not. Induced laser power is $10 \mathrm{~W}$ for total and laser profile is Gausiaan distribution with about $2.5 \mathrm{~mm}$ of half-length of half-maximum. Ignition delay time was nearly $0.3 \mathrm{~s}$ after the laser exposure under the considered conditions, although it has shown that the ignition delay time tended to longer when the condition is approached to lowest pressure to be ignited. Reproducibility is carefully checked by preliminary test in standard pressure condition, however, it is also true that error increases when the lower pressure is employed due to the system limitation, especially in low oxygen concentration regime. Ignition boundary near the lowest pressure with low oxygen content (in Fig. 1, P 35 kPa) is therefore questionable. For the other details, see Refs.6-8.
} 
phase and thermal degradation reactions in solid phase), and physical processes, including heat and mass transport, in gas/solid phases. In this study, ceiling-like sample orientation (the sample is irradiated from the bottom) is considered. Thus, buoyancy-driven flow may form the stagnation-point flow field below the surface to affect the ignition characters.

\section{Analytical Study}

At first, it is better to start with the analytical approach to understand the how the pressure affects on the ignition characters, if any. Although the most of analytical solutions are required numerous assumptions to simplify the problem, it could provide the dependency of all system parameters on interested issues in mathematical form and is valuable to apply in the first place.

Let us consider the ignition processes considered here are separated to two stages; these are, prior/after the time when the solid degradation reactions start. We can call the former as "inert" since we have no gaseous fuel matter which responsible for the ignition, whereas call the latter as "active". Since "inert" stage is essentially independent with pressure, a difference in the imposed pressure conditions may only appear on the "active" stage. Now our interest is replaced to the following: to look for the ignition induced by the heated fuel gas ejected into the cold oxidized, sub-atmospheric pressure environment. Apparently this is typical two-point boundary value problem in one-dimensional field. Concentrations of fuel and oxygen are nearly constant at 1.0 for fuel (pure fuel) and 0.21 for oxygen $\left(21 \% \mathrm{O}_{2}\right.$ as air) in each boundary, respectively.

In this study, two represented analytical models are considered to determine the gas phase ignition character above mentioned: one is the pure diffusion model proposed by Liňán \& Crespo [10] and the other is the ignition in stagnation-point flow field developed by Niioka [11] (see Fig.2). In Liňán \& Crespo's model, two gaseous reactants (i.e. fuel and oxidizer) meet at time zero and diffuse into each layer to form the mixture, then finally the mixture is spontaneously ignited at certain location. In Niioka's model, fuel and oxidizer flow are encountered to form the stagnation-point flow field and ignition is observed there. The main difference of these models is with/without the excess heat loss associated with the stretched flow field; it is additionally concerned in Niioka's model over Liňán \& Crespo's model. In the following, we may call these ignition modes as "(a) diffusion mode" and "(b) stretched mode", respectively (see (a)(b) in Fig.2).

(a) pure diffusion field

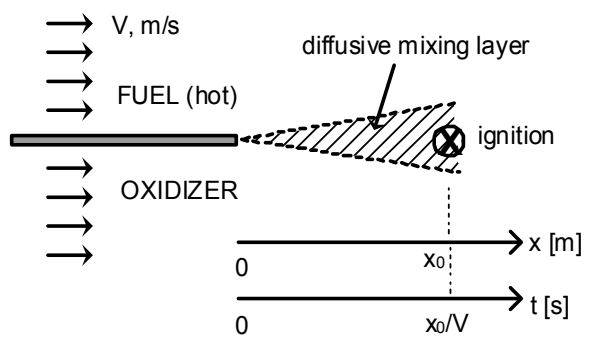

(b) stagnation point flow field

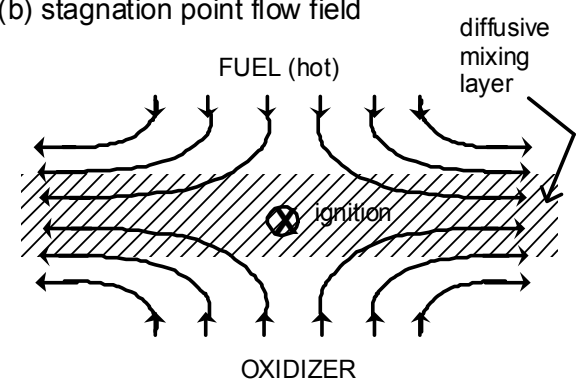

Fig. 2 Schematics of analytical model considered in this study [11]

\subsection{Analytical solution in pure diffusion field (Liňán \& Crespo’s model) [10]}

Based on the Liňán \& Crespo's model with asymptotic analysis, the following equation is given to predict the ignition character. Although several type of problems are concerned in their paper, the following equation is the one to express the ignition for different initial temperatures of the reactants: 


$$
\begin{aligned}
& -\frac{\xi^{2}}{\tau_{1}} \frac{\partial^{2} \phi}{\partial \xi^{2}}=(\xi-\beta \phi)^{b} \exp [\phi-\xi] \\
& \left(\begin{array}{l}
x=\frac{1-\operatorname{erf}(\eta)}{2}, \quad \eta=\frac{z}{2 \sqrt{\tau}}, \quad z=\left(\rho^{2} D t_{1}\right)^{-1 / 2} \int_{0}^{X} \rho d X \\
\xi=\beta_{1} x, \quad \tau_{1}=\frac{\tau}{\beta_{1}^{b} \eta_{1}^{2}}, \quad \tau=\frac{t}{t_{1}}
\end{array}\right) \\
& \phi \quad \text { : non-dimensional temperature increment } \\
& t_{1} \quad \text { : characteristic chemical time (constant) } \\
& X \quad: 1 \text {-D coordinate } \\
& a, b \quad: \text { reaction order (constant) } \\
& \eta_{1} \quad \text { : location of the reaction zone satisfying following relation; } \\
& \eta_{1}^{2}=\ln \left(\beta_{1} / 2 \sqrt{\pi}\right)-\ln \eta_{1} \\
& \beta_{1} \quad: \text { system parameter }\left\{\beta_{1}=E\left(T_{o x}-T_{f}\right) / R T_{o x}^{2}\right\} \\
& \beta \quad \text { : system parameter }\left\{\beta=c_{p}\left(T_{o x}-T_{f}\right) / Q Y_{o x, 0}\right\}
\end{aligned}
$$

where, $c_{p}, Q, \rho, D$ and $E$ denote specific heat capacity at constant pressure, heat of the reaction, density, mass diffusion coefficient and activation energy, respectively. $Y_{o x, 0}$, $T_{o x}$ and $T_{f}$ indicate the initial mass fraction of oxygen in oxidizer, initial temperature of oxidizer and fuel, respectively. Note that $\rho^{2} D$ is set to be constant to take into account the temperature- and pressure-dependency in $D$. In this expression, the ignition character, $\phi$, which is related to the delay time and limit is determined by two parameters of $\beta_{1}$ and $\beta$. More importantly, $\beta_{1}$ and $\beta$ are independent with the pressure and stay as constant in various pressure fields. Therefore, the reduction of pressure may not alter the ignition delay time. Ignition limit is actually controlled by the parameters of $\beta_{1}$ and $\beta$ and it contains only initial value of mass fractions of oxidizer. In this sense, the ignition limit is determined by not the partial pressure of oxygen, but the oxygen concentration, $Y_{o x, 0}$, in the oxidizer flow.

\subsection{Analytical solution in stagnation point flow field (Niioka's model) [11]}

Based on the Niioka's model with asymptotic analysis, on the other hand, the following equation is given to predict the ignition character. Note that the following equation is also the one to express the ignition for different initial temperatures of the reactants:

$$
\left.\begin{array}{l}
-\frac{\zeta^{2}}{\sigma} \frac{\partial^{2} \phi}{\partial \zeta^{2}}=(\zeta-\beta \phi) \exp [\phi-\zeta] \\
\zeta=\beta_{1} \xi^{\prime}, \quad \xi^{\prime}=\frac{1}{2} \operatorname{erfc}\left[\frac{\eta}{\sqrt{2\left(1-e^{-2 \tau}\right)}}\right], \quad \sigma=\left(1-e^{-2 \tau}\right) \frac{\Lambda_{1}}{2 \alpha \eta_{1}^{2}} \\
\Lambda_{1}=\frac{E^{*}}{\theta_{0}^{2}} D_{I} Y_{o x, 0} Y_{f, 0} \exp \left(-\frac{E^{*}}{\theta_{0}}\right), \quad D_{I}=\frac{A Q^{*} \rho n M_{o x}}{2 a}
\end{array}\right)
$$

where, $E^{*}, \theta_{0}, A, n, M_{o x}$ and $a$ denote non-dimensional activation energy, non-dimensional temperature, frequency factor (constant), stoichiometric coefficient, molecular weight of oxidizer and velocity gradient (stretch rate), respectively. $Y_{f, 0}$ indicates the initial mass fraction of fuel in fuel flow. It is similar to what given in Liňán \& Crespo's solution (Eq.(1)), but slight difference can be seen in the system-controlling parameter. In eq.(2), an ignition character; such as $\phi$, is also determined by two parameters of $\sigma$ and $\beta$. Important to note is that $\sigma$ includes the contribution from 
pressure and velocity. Thus, the reduction of pressure could alter the ignition delay time. Considering that the fuel is fixed; i.e. $n, Q^{*}$ (non-dimensional expression of $Q$ ) and $A$ stay as constants, the ignition limit given by the parameter $\Lambda_{1}$ could be determined by $\rho Y_{o x, 0} Y_{f, 0}$. In this sense, the ignition limit is determined by not the oxygen concentration, but the partial pressure of oxygen, $\rho Y_{o x, 0}\left(\sim p_{o x, 0}\right)$, in the oxidizer flow (just opposite to Liňán \& Crespo's solution).

As noted above two models, the pressure dependency on the ignition limit may appear or vanish, depending on with/without the convection-associated heat loss. In other word, the pressure may play a role on the ignition criteria depending on the flow field formed over the possible ignitable zone. Expected ignition-limit curve in oxygen-pressure plane (see Fig.1(b)) given by two theories are schematically summarized in Fig.3. For comparison purpose, a corresponding curve obtained by our previous experiment is embedded.

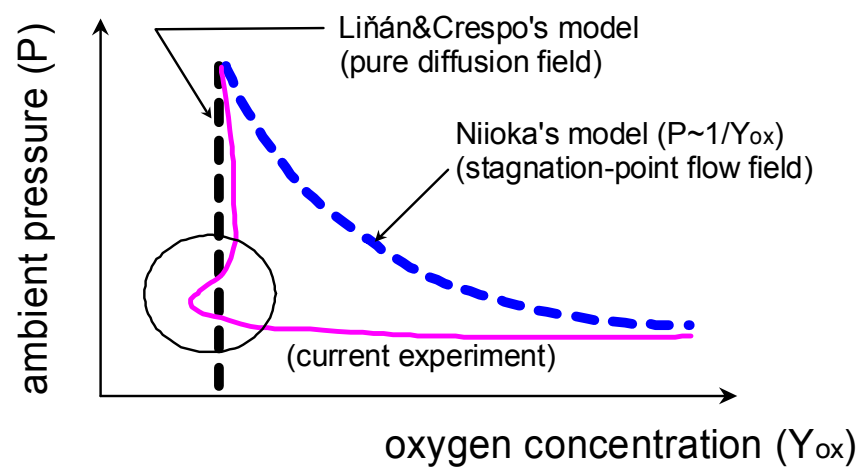

Fig. 3 Schematical illistration of suggested ignition boundaries given by two classical theories (Liňán\&Crespo's model [10] and Niioka's model [11])

As shown in the figure, the experimentally-obtained limit curve is rather close to the one predicted by Liňán \& Crespo's model in the middle pressure range, then it sharply approaches to the one predicted by Niioka's model when the imposed pressure is very close to the critical value to be ignited. The trend of measured data near the lowest pressure in low oxygen concentration (see circle in Fig.3) is somewhat unpredictable via aforementioned two theoretical models. Although it might be due to chemical effects (e.g. radical recombination or surface quenching), it also may come from the experimental error as stated previously. Thus, further argument on this part would not be made here at this moment. Now the main question is why the measured data would rather follow to Liňán \& Crespo's model (diffusion mode), not to Niioka's model (stretched mode) in the wide range of sub-atmospheric pressure. Looking back to our experiment [6]-[8], the hot fuel is ejected to downward whereas the heated gas oppositely moves upward to the fuel surface due to the buoyancy. The upward flow speed driven by buoyancy increases as the time elapsed. In this sense, it is suspected that the real case could be categorized to either pure diffusion or stagnation-point flow condition depending on the ignition delay time. For example, if the ignition delay time is very short, then the buoyancy-driven convective transport hardly plays a role on it. As consequent, the pure diffusion model could be applied to predict the ignition character in such case. On the contrary, if the delay time is relatively longer for the buoyancy-driven flow enough to be developed, the stagnation-point flow model is more reasonable to apply. Although the precise ignition delay time was not measured in previous works [6]-[8], it has been notified that the delay time under the condition along the ignition-limit curve was nearly constant in middle pressure range (40 kPa $100 \mathrm{kPa}$ ), but became longer in lower pressure [7]. This fact may support above 
speculation. Next, we shall verify above speculation via 3-D numerical simulation.

\section{Numerical Study}

As denoted previously, above analytical solutions are too identical; for example the flow field is fixed entire event although the real case it is, obviously, varied with time due to the buoyancy. Here, 3-D numerical calculation with time-dependent mass and heat transfer processes including one-step gas/solid phases chemical reactions is performed to see how the flow field is formed below the surface prior to the ignition. Note that only the qualitative trend is of interest, therefore, any quantitative discussion is not made here.

\subsection{Description of Applied Numerical Model}

Simulation of irradiated ignition processes is performed by FDS (Fire Dynamic Simulator) [12] originally developed at NIST (The National Institute of Standards and Technology). Since the original code does not include the precise solid combustion module which applicable for the present purpose, corresponding one is developed by author and added to the original set. Its validity and the details can be found in elsewhere [13, 14]. Applied numerical model is shown in Fig.4. Thin cellulose sheet considered as the solid fuel is placed at the top of the domain and is heated by external radiation from the bottom. Gaussian profile of thermal radiation is assumed (half-radius half-maximumis is $2.5 \mathrm{~mm}$ ) to make consistent with the corresponding experiment; such as laser heating. The decomposed gaseous products, including both combustible and inert components, are assumed as transparent and do not absorb the incident radiation. To take advantage of the symmetry of the phenomena, only half volume is considered as the numerical domain. Two represented pressure conditions, such as "standard" (101 kPa) and "reduced (sub-atmospheric)" (40 kPa) pressure, are shown for comparison purpose.

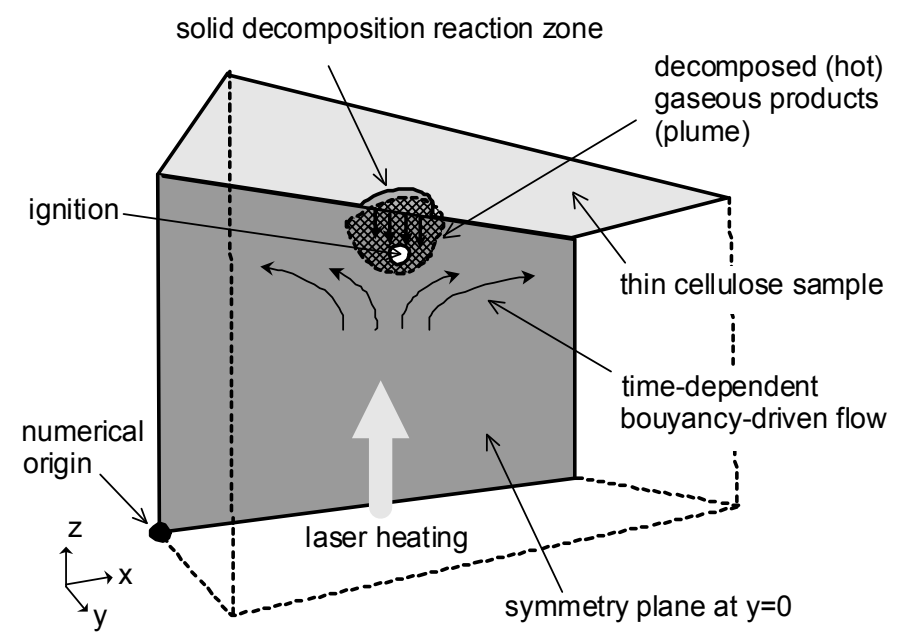

Fig. 4 Schematical numerical model for 3-D numerical simulation

\subsection{Numerical Results}

In Fig.5, time changes of (a) mass fraction of fuel [-], (b) heat release rate $\left[\mathrm{GW} / \mathrm{m}^{3}\right]$, (c) velocity in $\mathrm{z}$-direction (i.e. normal to the surface) $[\mathrm{m} / \mathrm{s}]$ in "standard" pressure $(101 \mathrm{kPa})$ are shown. Magnified velocity vector plot formed just prior to the ignition is added in (d). Positive (negative) sign of velocity component reveals that the flow direction is upward (downward). To indicate how the solid is heated during the ignition event, surface temperature contour is superimposed in all figures. 
(a) mass fraction of fuel gas (range: $\mathrm{Yf}=0.0 \sim 0.05[-]$ )
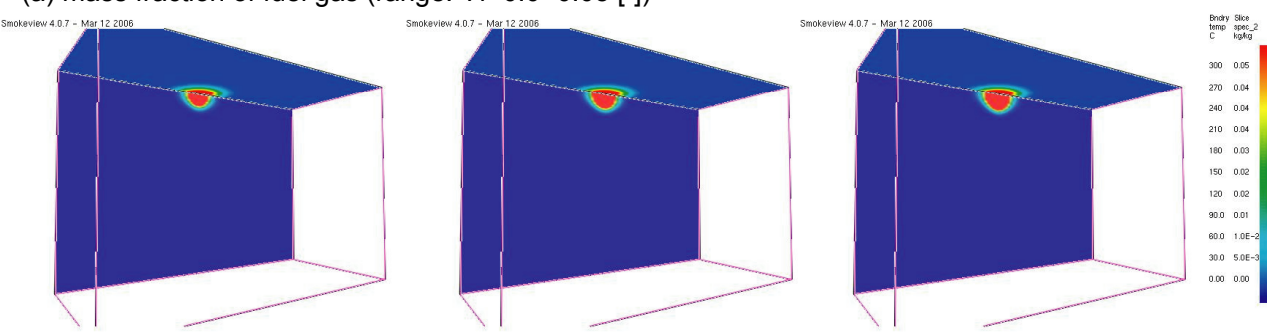

(b) heat release rate (range: $\mathrm{Q}=0.0 \sim 6.5 \times 10^{5}\left[\mathrm{~kW} / \mathrm{m}^{3}\right]$ )
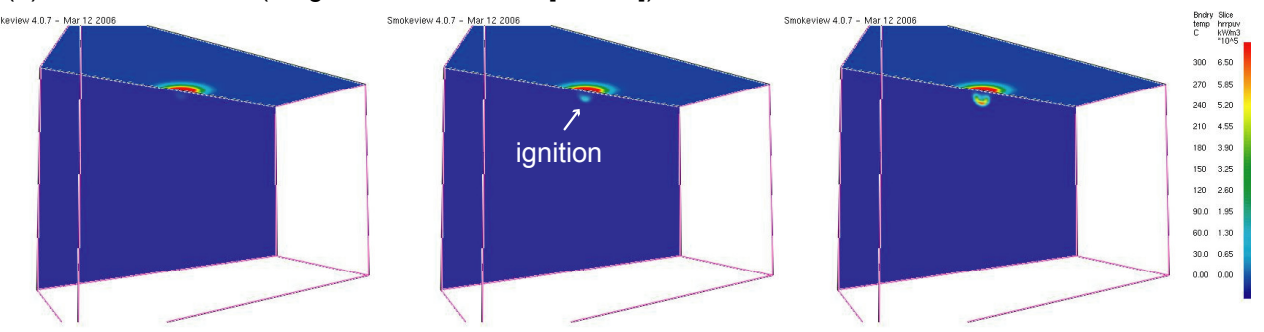

(c) z-direction velocity (range: $v=-0.5 \sim 0.2[\mathrm{~m} / \mathrm{s}]$ )
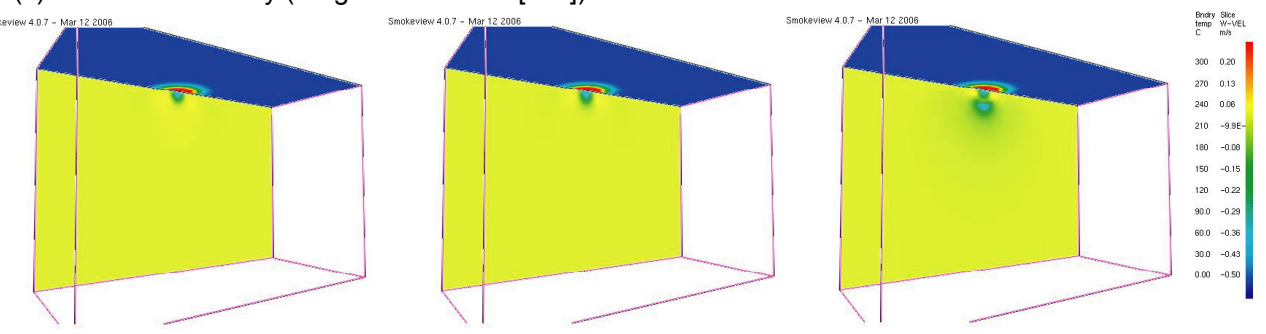

(d) velocity vector plot at $\mathrm{t}=0.360 \mathrm{~s}$ (just before ignition)

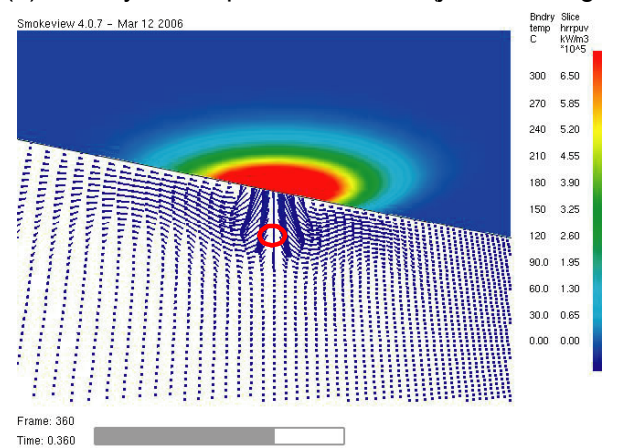

conditions: $\mathrm{P}=101 \mathrm{kPa}, \mathrm{Y}_{\mathrm{ox}, 0}=0.5$ time (from left to right in (a)-(c)): $\mathrm{t}=0.360,0.366,0.368 \mathrm{~s}$

Fig. 5 Contour plots of calculated physical properties at $y=0$ plane: (a) mass fraction of fuel, (b) heat release rate, (c) velocity in $\mathrm{z}$-direction, (d) magnified velocity vector plot at $t=0.360 \mathrm{~s}$. From the left to right, corresponding time is $\mathbf{t}=\mathbf{0 . 3 6 0}, \mathbf{0 . 3 6 6 , 0 . 3 6 8} \mathrm{s}$ after the irradiation, respectively, in (a)-(c).

Circle in (d) indicates the ignition location. Imposed pressure is at standard condition $(\mathrm{P}=101 \mathrm{kPa})$.

As indicated in Fig.5, an ignition event starts from the location where very close to the surface (see (b)), suggesting that the heated surface plays a role on the initiation of the slightly inside the fuel plume edge. Note that the red color of mass fraction of the fuel corresponds to 0.05 and the edge of it corresponds to the zone of combustible mixture (co-existing zone with fuel and oxygen). In (c), it is seen that velocity in z-direction around the irradiation center axis shows always negative, that is, the flow is pointed to downward. This fact implies that the ignition occurs during the solid degradation stage and, more importantly, the buoyancy-induced flow is not fully developed onset of the ignition observed here. Magnified image in (d) also shows little encountered flow to induce the stretched-associated heat loss. Thus, the ignition observed here can be described by Liňán \& Crespo's model (diffusion mode). 
(a) mass fraction of fuel gas (range: $Y f=0.0 \sim 0.05[-]$ )
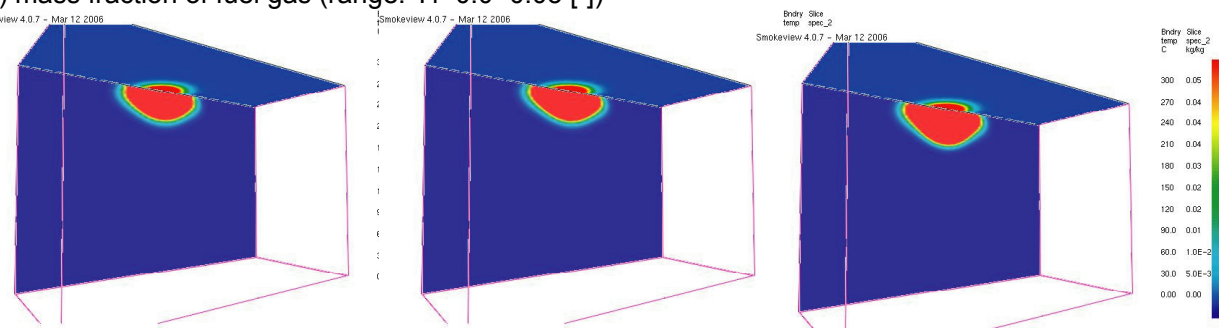

(b) heat release rate (range: $\mathrm{Q}=0.0 \sim 3.0 \times 10^{5}\left[\mathrm{~kW} / \mathrm{m}^{3}\right]$ )
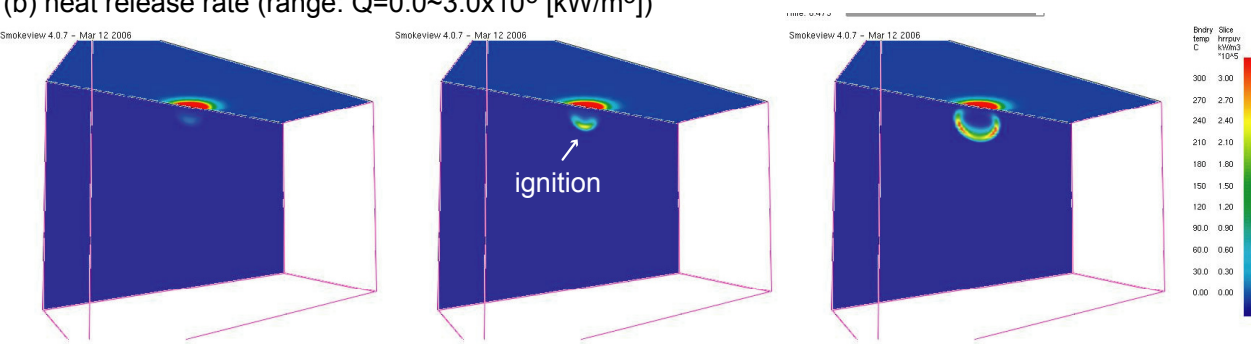

(c) z-direction velocity (range: $v=-0.5 \sim 0.2[\mathrm{~m} / \mathrm{s}]$ )
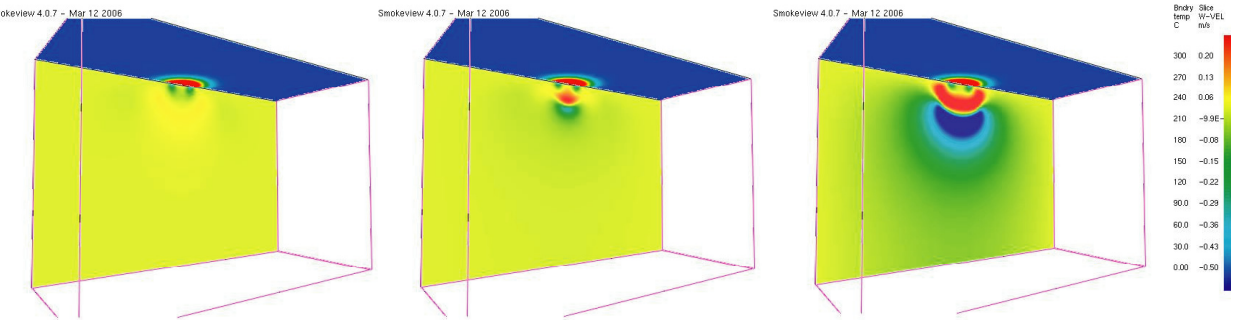

(d) velocity vector plot at $\mathrm{t}=0.472 \mathrm{~s}$ (just before ignition)

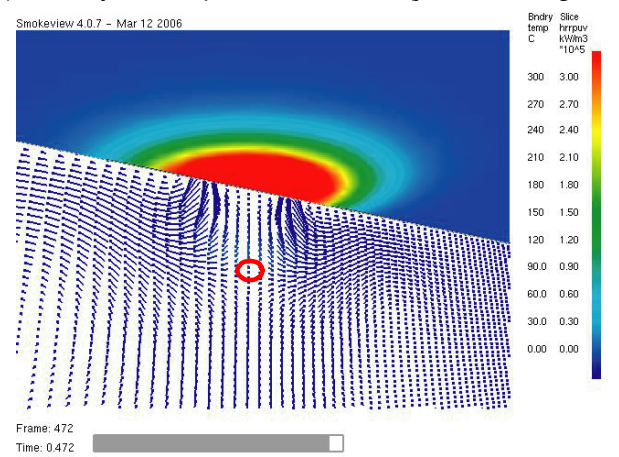

conditions: $\mathrm{P}=40 \mathrm{kPa}, \mathrm{Y}_{\mathrm{ox}, 0}=0.5$ time (from left to right in (a)-(c)): $\mathrm{t}=0.472,0.477,0.479 \mathrm{~s}$

Fig. 6 Contour plots of calculated physical properties at $y=0$ plane: (a) mass fraction of fuel, (b) heat release rate, (c) velocity component of $z$-direction, (d) magnified velocity vector plot at $t=0.472 \mathrm{~s}$.

From the left to right, corresponding time is $\mathbf{t}=\mathbf{0 . 4 7 2}, 0.477,0.479 \mathrm{~s}$ after the irradiation, respectively, in (a)-(c). Circle in (d) indicates the ignition location. Imposed pressure is in sub-atmospheric range $(\mathrm{P}=40 \mathrm{kPa})$.

In Fig.6, on the other hand, calculated results in "reduced" pressure (40 kPa) are shown. Since the ignition delay time $(0.477 \mathrm{~s})$ is longer than that what is obtained in Fig.5 (0.366 s), more fuel is ejected and larger fuel plume is formed under the irradiated surface (see (a)). Lager hot plume might be also because the pressure effect; diffusion coefficient, $D$, is inversely correlated to the pressure and diffusive transport is relatively pronounced in low pressure. One important to note is that the ignition location, which is obviously penetrated in the fuel plume as compared to what is seen in Fig.5. Moreover, apparent difference is found in the flow field at that location: only downward flow field is formed in Fig.5, whereas encountered flow field, i.e. stagnation-point flow field, is clearly observed in Fig.6 (see (d)), just prior to the ignition. This is, of course, due to the buoyancy-induced flow. In addition, the degradation reactions in the solid at the irradiated center are nearly ceased 
and little inertia is expected toward the downward. Therefore, the mixture is pushed back to the hot surface to increase the chance to be ignited. After all, it is known that the ignition observed in Fig. 6 could be better described by Niioka's model (stretched mode), rather than the Liňán \& Crespo's model (diffusion mode).

As summarized above, it is confirmed that the ignition mode is strongly related to an ignition delay time in natural convection system and both modes appeared in pure diffusion and stagnation-point flow field could be observed depended on the imposed conditions. Ignition showed in our experiment would be categorized to pure diffusion mode, suggesting that the convective transport has little effect on ignition processes. When the convective process comes to play a role, the ignition mode would be approached to what would be attained in stagnation-point flow field, rather than the pure diffusion field. Under such condition, even in sub-atmospheric pressure, ignition-limit curve (as seen in Fig.1) in pressure and oxygen plane will be followed by $p \propto Y_{o x, 0}^{-1}$, suggesting that critical $\mathrm{ppO}_{2}$ can be defined to see if the ignition can occur or not. According to our experiment, it is notified that the ignition delay time tends to be longer in low pressure, this supports the ignition may change from "diffusion mode" to "stretched mode". To observe this transition more clearly, slower heating ignition test $(<10 \mathrm{~W})$ would be one good candidate since it can increase the delay time. We now plan to perform such series of experiment to confirm our theoretical and numerical predictions.

\section{Concluding Remarks}

A change in criterion of irradiated ignitable regime under low pressure is investigated by using the classical ignition theories and 3-D numerical simulation. Either pure diffusion mode or convective heat loss mode is considered and the currently-obtained ignition character by author's group is likely categorized to the former one: there would be little convective (stretched) heat loss effect on ignition. It is suspected that weaker irradiative power as ignition source could induce longer ignition delay time and the buoyancy-induced flow comes to play on ignition processes. These modes are properly predicted in time-dependent 3-D simulation at which the flow field in natural convection system varies with time. Nonetheless, two classical ignition theories show two-extreme cases, it turns out that they are quite helpful to consider what kind of physics lies on the observed ignition in low pressure.

\section{Acknowledgments}

This work is partially supported by Grants-in-Aid for Young Scientists (B) of \#19710140, The Kurata Memorial Hitachi Science and Technology Foundation and Japan Society for the Promotion of Science. Ms. Aoki, former graduate student, and Mr. Kusano, former undergraduate student, gave a dedicated help in the experimental part. Author would like to express sincere thank for their supports and contributions.

\section{References}

(1) http://www.nasa.gov/missions/solarsystem/cev.html

(2) Saito, T., Harayama, M. and Iwama, A., Ignition of Composite Propellant Composed of Polybutadiene and Ammonium Perchlorate by means of Carbon Dioxide Laser at Subatmospheric Pressure (I): Ignition Behaviors in Combustion Supporting, Non-combustion Supporting, and Inert Gas Environments under Low Radiative Heat Flux, J. Ind. Explosives Society of Japan 41(3), 1980, 131-140 (in Japanese).

(3) Saito, T., Harayama, M. and Iwama, A., Ignition of Composite Propellant Composed of Carboxyl-terminated Polybutadiene and Ammonium Perchlorate at Subatmospheric Pressure by means of Carbon Dioxide Laser (II): Drawing Up the Ignition Characteristic Maps in Helium, Argon, and Oxygen Environments and Discussion on Pulsating Ignition 
Phenomena, J. Ind. Explosives Society of Japan 42(1), 1981, 17-23 (in Japanese).

(4) Yang, J.C., Hamins, A. and Donnelly, M.K., Reduced Gravity Combustion of Thermoplastic Spheres, Combust. Flame 120, 2000, 61-74.

(5) Goldmeer, J.S., T'ien, J.S. and Urban, D.L., Combustion and Extinction of PMMA Cylinders during Depressurization in Low-gravity, Fire Safety J. 32, 1999, 61-88.

(6) Nakamura, Y. and Aoki, A., Ignition of Solid Fuels at Low Pressure, Selected Paper presented at 25th Int'l. Sympo. on Space Tech. Sci. (ISTS), Kanazawa Japan, 2006, 922-927.

(7) Nakamura, Y. and Aoki, A., Irradiated Ignition of Solid Materials in Reduced Pressure Atmosphere with Various Oxygen Concentrations -For Fire Safety in Space Habitats-, Adv. Space Res., in print.

(8) Nakamura, Y., Aoki, A., Fujita, O. and Ito, H., Similarity in Irradiated Ignition Characteristics of Cellulosic Paper under Low Gravity and Low Pressure Environments, Proc. 5th Int'l. Sympo. Scale Modeling (ISSM5), Choshi Chiba, Sept. 2006, 89-98.

(9) Nakamura, Y., Irradiated Ignition of Solid Combustibles: Application to a Fundamental Fire Research, J. Combust. Soc. Japan 48-146, 2006, 328-334. (in Japanese).

(10) Liňán, A. and Crespo, A., An Asymptotic Analysis of Unsteady Diffusion Flames for Large Activation Energies, Combust. Sci. Tech. 14, 1976, 95-117.

(11) Niioka, T., Ignition Time in the Stretched Flow Field, Proc. Combust. Inst. 18, 1981, 1807-1813.

(12) http://fire.nist.gov/fds/

(13) Nakamura, Y., Kashiwagi, T., McGrattan, K.B. and Baum, H.R., Enclosure Effects on Flame Spread over Solid Fuels in Microgravity, Combust. Flame 130, 2002, 307-321.

(14) Nakamura, Y. and Kashiwagi, T., Study of Sample Orientation on Non-Piloted Ignition of Thin PMMA Sheet by Laser- Part.1 Theoretical Prediction, Combust. Flame 141, 2005, 149-169. 